\title{
Common Fixed Point Theorem for Weakly Compatible Maps in Intuitionistic Fuzzy Metric Spaces using Implicit Relation
}

\author{
Saurabh Manro* \\ School of Mathematics and Computer Applications, Thapar University, \\ Patiala, Punjab \\ *Email: sauravmanro@yahoo.com
}

\begin{abstract}
In this paper, we use the notion of property E.A. in an intuitionistic fuzzy metric space to prove a common fixed point theorem which generalizes Theorem-2 of Turkoglu et al. (2006).
\end{abstract}

Key words: Intuitionistic fuzzy metric space, property E.A, implicit relation.

Subject classification: 2001 AMS: 47H10, 54H25

\section{INTRODUCTION}

I n 1986, Jungck introduced the notion of compatible maps for a pair of self mappings. Several papers involving compatible maps proved the existence Lof common fixed points in the classical and fuzzy metric spaces (Grorge and Veeramani, 1994, Kramosil and Michalek, 1975). Aamri and Moutawakil (2002) generalized the concept of non compatibility by defining the notion of property E.A. and proved common fixed point theorems under strict contractive conditions. Atanassove (1986) introduced and studied the concept of intuitionistic fuzzy sets as a generalization of fuzzy sets and later there has been much progress in the study of intuitionistic fuzzy sets by many authors (Alaca, 2006; Atanassov, 1986; Coker, 1997; Manro et al., 2010, 2012; Park, 2004; Park et al. 2005; Saadati and Park, 2006). In 2004, Park defined the notion of intuitionistic fuzzy metric space with the help of continuous $t$-norms and continuous $t$-conorms as a generalization of fuzzy metric space due to George and Veeramani (1994). Fixed point theory has important applications in diverse disciplines of mathematics, statistics, engineering, and economics in dealing with problems arising in: Approximation theory, potential theory, game theory, mathematical economics, etc. Several authors (George and Veeramani 1994; Kramosil and Michalek, 1975) proved some fixed point theorems for various generalizations of contraction mappings in probabilistic and fuzzy metric space. Turkoglu et al. (2006) gave a generalization of Jungck's common fixed point theorem (Jungck, 1976) to intuitionistic fuzzy metric spaces. In this paper, we

\footnotetext{
Mathematical Journal of Interdisciplinary Sciences Vol. 2, No. 2, March 2014 pp. 209-218

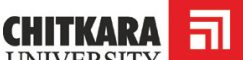
UNIVERSITY

C 2014 by Chitkara University. All Rights Reserved.
} 
Manro, S.

use the notion of property E.A. in an intuitionistic fuzzy metric space to prove a common fixed point theorem for a quadruplet of self mappings in intuitionistic fuzzy metric space.

\section{PRELIMINARIES.}

The concepts of triangular norms $(t-$ norm $)$ and triangular conorms $(t-$ conorm) are were originally introduced by Schweizer and Sklar (1960) in the study of statistical metric spaces.

Definition 2.1. (Schweizer and Sklar, 1960) A binary operation *: $[0,1] \times$ $[0,1] \rightarrow[0,1]$ is continuous $t$-norm if $*$ satisfies the following conditions:

(i) * is commutative and associative;

(ii) * is continuous;

(iii) $\quad a^{*} 1=a$ for all $\mathrm{a} \in[0,1]$;

(iv) $a * b \leq c * d$ whenever $a \leq c$ and $b \leq d$ for all $a, b, c, d \in[0,1]$.

Definition 2.2. (Schweizer and Sklar, 1960) A binary operation $\diamond$ : $[0,1] \times$ $[0,1] \rightarrow[0,1]$ is continuous $t$-conorm if $\diamond$ satisfies the following conditions:

(i) $\diamond$ is commutative and associative;

(ii) $\diamond$ is continuous;

(iii) $a \diamond 0=a$ for all $a \in[0,1]$;

(iv) $a \diamond b \leq \mathrm{c} \diamond \mathrm{d}$ whenever $a \leq c$ and $b \leq d$ for all $a, b, c, d \in[0,1]$.

Alaca et al. (2006) using the idea of Intuitionistic fuzzy sets, defined the notion of intuitionistic fuzzy metric space with the help of continuous $t$-norm and continuous $t$ - conorms as a generalization of fuzzy metric space due to Kramosil and Michalek (1975) as :

Definition 2.3. (Alaca et al., 2006) A 5-tuple $(X, M, N, *, \diamond)$ is said to be an intuitionistic fuzzy metric space if $X$ is an arbitrary set, $*$ is a continuous $t$-norm, $\diamond$ is a continuous $t$-conorm and $M, N$ are fuzzy sets on $X^{2} \times[0, \infty)$ satisfying the following conditions:

(i) $\quad M(x, y, t)+N(x, y, t) \leq 1$ for all $x, y \in X$ and $t>0$;

(ii) $M(x, y, 0)=0$ for all $x, y \in X$;

(iii) $M(x, y, t)=1$ for all $x, y \in X$ and $t>0$ if and only if $x=y$;

(iv) $M(x, y, t)=M(y, x, t)$ for all $x, y \in X$ and $t>0$;

(v) $M(x, y, t) * M(y, z, s) \leq M(x, z, t+s)$ for all $x, y, z \in X$ and $s, t>0$; 
(vi) for all $x, y \in X, M(x, y,):.[0, \infty) \rightarrow[0,1]$ is left continuous;

(vii) $\lim _{n \rightarrow \infty} M(x, y, t)=1$ for all $x, y \in X$ and $t>0$;

(viii) $N(x, y, 0)=1$ for all $x, y \in X$;

(ix) $N(x, y, t)=0$ for all $x, y \in X$ and $t>0$ if and only if $x=y$;

(x) $\quad N(x, y, t)=N(y, x, t)$ for all $x, y \in X$ and $t>0$;

(xi) $\quad N(x, y, t) \diamond N(y, z, s) \geq N(x, z, t+s)$ for all $x, y, z \in X$ and $s, t>0$;

(xii) for all $x, y \in X, N(x, y,):.[0, \infty) \rightarrow[0,1]$ is right continuous;

(xiii) $\lim _{n \rightarrow \infty} N(x, y, t)=0$ for all $x, y \in X$.

Then $(M, N)$ is called an intuitionistic fuzzy metric space on $X$. The functions $M(x, y, t)$ and $N(x, y, t)$ denote the degree of nearness and the degree of non-nearness between $x$ and $y$ w.r.t. $t$ respectively.

Remark 2.1.(Alaca et al., 2006) Every fuzzy metric space $(X, M, *)$ is an intuitionistic fuzzy metric space of the form $(X, M, 1-M, *, \diamond)$ such that $t$-norm $*$ and $t$-conorm $\diamond$ are associated as

$$
x \diamond y=1-\left((1-x)^{*}(1-y)\right) \text { for all } x, y \in X
$$

Remark 2.2.(Alaca et al., 2006) In intuitionistic fuzzy metric space $(X, M$, $N, *, \diamond), M(x, y,$.$) is non-decreasing and N(x, y,$.$) is non-increasing for all x$, $y \in X$.

Alaca et al. (2006) introduced the following notions:

Definition 2.4. Let $(X, M, N, *, \diamond)$ be an intuitionistic fuzzy metric space. Then

(a) a sequence $\left\{x_{n}\right\}$ in $X$ is said to be Cauchy sequence if, for all $t>0$ and $p>0$,

$$
\lim _{\mathrm{n} \rightarrow \infty} M\left(x_{n+p}, x_{n}, t\right)=1 \text { and } \lim _{\mathrm{n} \rightarrow \infty} N\left(x_{n+p}, x_{n}, t\right)=0
$$

(b) a sequence $\left\{x_{n}\right\}$ in $X$ is said to be convergent to a point $x \in X$ if, for all $\mathrm{t}>0$,

$$
\lim _{\mathrm{n} \rightarrow \infty} M\left(x_{n}, x, t\right)=1 \text { and } \lim _{\mathrm{n} \rightarrow \infty} N\left(x_{n}, x, t\right)=0
$$

Definition 2.5.(Alaca et al., 2006) An intuitionistic fuzzy metric space $(X, M$, $N, *, \diamond)$ is said to be complete if and only if every Cauchy sequence in $X$ is convergent.

Example 2.1.(Alaca et al., 2006) Let $X=\left\{\frac{1}{n}: n=1,2,3, \ldots\right\} \cup\{0\}$ and let $*$ be the continuous $t$-norm and $\diamond$ be the continuous $t$-conorm defined by $a * b=a b$
Common Fixed

Point Theorem for Weakly Compatible Maps in Intuitionistic Fuzzy Metric Spaces using Implicit Relation 
Manro, S.

and $a \diamond b=\min \{1, a+b\}$ respectively, for all $a, b \in[0,1]$. For each $t \in(0$, $\infty)$ and $x, y \in X$, define $(M, N)$ by

$$
M(x, y, t)=\left\{\begin{array}{c}
\frac{t}{t+|x-y|}, t>0, \\
0, \quad t=0
\end{array} \quad \text { and } N(x, y, t)=\left\{\begin{array}{c}
\frac{|x-y|}{t+|x-y|}, t>0 \\
1, \quad t=0 .
\end{array}\right.\right.
$$

Clearly, $(X, M, N, *, \diamond)$ is complete intuitionistic fuzzy metric space.

Definition 2.6. A pair of self mappings $(f, g)$ of an intuitionistic fuzzy metric space $(X, M, N, *, \diamond)$ is said to be commuting if

$$
M(f g x, g f x, t)=1 \text { and } N(f g x, g f x, t)=0 \text { for all } x \in X
$$

Definition 2.7. (Aamri and Moutawakil, 2002) A pair of self mappings ( $f$, $g$ ) of an intuitionistic fuzzy metric space $(X, M, N, *, \nabla)$ is said to satisfy the property $E$.A if there exist a sequence $\left\{x_{n}\right\}$ in $X$ such that $\lim _{n \rightarrow \alpha} M\left(f x_{n}, g x_{n}, t\right)=$ 1 and $\lim _{n \rightarrow \infty} N\left(f x_{n}, g x_{n}, t\right)=0$.

Example 2.2.(Aamri and Moutawakil, 2002) Let $X=[0, \infty)$. Consider $(X$, $\left.M, N,{ }^{*}, \diamond\right)$ be an intuitionistic fuzzy metric space as in Example 2.1. Define $f, g: X \rightarrow X$ by $f x=\frac{x}{5}$ and $g x=\frac{2 x}{5}$ for all $x \in X$. Then for sequence $\left\{x_{n}\right\}=\left\{\frac{1}{n}\right\}$ , $\lim _{\mathrm{n} \rightarrow \infty} M\left(f x_{n}, g x_{n}, t\right)=1$ and $\lim _{\mathrm{n} \rightarrow \infty} N\left(f x_{n}, g x_{n}, t\right)=0$. Then $f$ and $g$ satisfy property E.A.

Definition 2.8.(Alaca et al., 2006) A pair of self mappings ( $f, g)$ of an intuitionistic fuzzy metric space $(X, M, N, *, \diamond)$ is said to be weakly compatible if they commute at coincidence points i.e. if $f u=g u$ for some $u \in X$, then $f g u$ $=g f u$.

It is easy to see that two compatible maps are weakly compatible.

Lemma 2.1.(Alaca et al., 2006) Let $(X, M, N, *, \diamond)$ be intuitionistic fuzzy metric space and for all $x, y \in X, t>0$ and if for a number $k \in(0,1)$,

$$
M(x, y, k t) \geq M(x, y, t)
$$

and

$$
N(x, y, k t) \leq N(x, y, t)
$$

then $x=y$. 


\section{MAIN RESULTS}

Turkoglu et al. (2006) proved the following Theorem:

Theorem 3.1. Let $(X, M, N, *, \diamond)$ be a complete intuitionistic fuzzy metric space. Let $f$ and $g$ be self mappings of $X$ satisfying the following conditions:

(a) $\quad g(X) \subseteq f(X)$;

(b) there exist $0<k<1$ such that

$$
M(g x, g y, k t) \geq M(f x, f y, t)
$$

and

$$
N(g x, g y, k t) \leq N(f x, f y, t)
$$

(c) $f$ is continuous.

Then $f$ and $g$ have a unique common fixed point provided $f$ and $g$ commute.

Now, we prove a common fixed point theorem using property E.A. in an intuitionistic fuzzy metric space, which is a generalization of Theorem 3.1 in the following way:

(i) to relax the continuity requirement of maps completely,

(ii) property E.A buys containment of ranges.

Theorem 3.2. Let $\left(X, M, N,{ }^{*}, \diamond\right)$ be an intuitionistic fuzzy metric space with continuous $t$-norm and continuous $t$ - conorm defined by $a * a \geq a$ and (1a) $\diamond(1-a) \leq(1-a)$ where $a \in[0,1]$. Let $f$ and $g$ be two weakly compatible self mappings of $X$ satisfying the following conditions:

(3.1) $f$ and $g$ satisfy the property E.A.;

(3.2) for each $x, y \in X, t>0$, there exist $0<k<1$ such that

$$
M(g x, g y, k t) \geq M(f x, f y, t)
$$

and

$$
N(g x, g y, k t) \leq N(f x, f y, t) ;
$$

(3.3) $f(X)$ or $g(X)$ is complete subspace of $X$.

Then $f$ and $g$ have a unique common fixed point.

Proof: In view of (3.1), there exists a sequence $\left\{x_{n}\right\}$ in $X$ such that $\lim _{n \rightarrow \infty} g x_{n}$ $=\lim _{\mathrm{n} \rightarrow \infty} f x_{n}=u$ for some $u \in X$. Suppose that $f(X)$ is complete subspace of $X$, 
Manro, S.

therefore, every convergent sequence of points of $f(X)$ has a limit point in $f(X)$ implies $\lim _{\mathrm{n} \rightarrow \infty} f x_{n}=f a=u=\lim _{\mathrm{n} \rightarrow \infty} g x_{n}$, for some $a \in X$, which implies that $u=f a \in f(X)$.

Now, we prove that $g a=f a$.

From (3.2) take $x=x_{n}, y=a$, we get

and

$$
M\left(g x_{n}, g a, k t\right) \geq M\left(f x_{n}, f a, t\right)
$$

$$
N\left(g x_{n}, g a, k t\right) \leq N\left(f x_{n}, f a, t\right) .
$$

Taking limit $n \rightarrow \infty$ on both sides, we get

$$
M(f a, g a, k t) \geq M(f a, f a, t)
$$

and

$$
N(f a, g a, k t) \leq N(f a, f a, t) .
$$

This implies by Lemma 2.1, $f a=g a$.

Therefore, $u=f a=g a$.

This shows that ' $a$ ' is coincident point of $g$ and $f$.

As $g$ and $f$ are weakly compatible, therefore, $g f a=f g a=f f a=g g a$.

Now, we show that $g a$ is the common fixed point of $g$ and $f$.

From (3.2) take $x=a, y=g a$,

$$
\begin{gathered}
M(g a, g g a, k t) \geq M(f a, f g a, t) \\
M(g a, g g a, k t) \geq M(g a, g g a, t)
\end{gathered}
$$

and

$$
\begin{gathered}
N(g a, g g a, k t) \leq N(f a, f g a, t), \\
N(g a, g g a, k t) \leq N(g a, g g a, t),
\end{gathered}
$$

This implies by Lemma 2.1, gga= $g a=f g a$.

This proves that $g a$ is the common fixed point of $g$ and $f$.

Now, we prove the uniqueness of common fixed point of $g$ and $f$. If possible, let $x_{0}$ and $y_{0}$ be two common fixed points of $f$ and $g$. Then by condition (3.2),

$$
M\left(x_{0}, y_{0}, k t\right)=M\left(g x_{0}, g y_{0}, k t\right) \geq M\left(f x_{0}, f y_{0}, t\right)=M\left(x_{0}, y_{0}, t\right)
$$

and

$$
N\left(x_{0}, y_{0}, k t\right)=N\left(g x_{0}, g y_{0}, k t\right) \leq N\left(f x_{0}, f y_{0}, t\right)=N\left(x_{0}, y_{0}, t\right) .
$$

Then by Lemma 2.1, we have $x_{0}=y_{0}$. 
Therefore, the mappings $f$ and $g$ have a unique common fixed point. This completes the proof.

Example 3.1. Let $X=\left\{\frac{1}{n}: n=1,2,3, \ldots\right\} \cup\{0\}$. Consider $(X, M, N, *, \diamond)$ be an intuitionistic fuzzy metric space as in Example 2.1. Define $f x=\frac{x}{4}$ and $g x=\frac{x}{12}$ for all $x \in X$. Clearly, $f$ and $g$ are weakly compatible mappings on $X$, Also,

(i) $f$ and $g$ satisfy the property E.A for the sequence $\left\{x_{n}\right\}=\left\{\frac{1}{n}\right\}$,

(ii) for $k=\frac{1}{3}$, the condition (3.2) of above theorem is satisfied,

(iii) $f(X)$ is complete subspace of $X$.

Thus all the conditions of Theorem 3.2 are satisfied and so $f$ and $g$ have the common fixed point $x=0$.

Theorem 3.3. Let $(X, M, N, *, \diamond)$ be an intuitionistic fuzzy metric space with continuous $t$-norm and continuous $t$ - conorm defined by $a * a \geq a$ and $(1-a)$ $\diamond(1-a) \leq(1-a)$, where $a$ in $[0,1]$. Let $f$ and $g$ be two weakly compatible self mappings of $X$ satisfying (3.1) and the following conditions:

(3.4) for each $x, y \in X, t>0$, there exist $0<k<1$ such that

$$
\begin{gathered}
M(f x, f y, k t) \geq \varphi(M(g x, g y, t), M(f x, g x, t), M(f y, g y, t), \\
M(f x, g y, t), M(f y, g x, t))
\end{gathered}
$$

and

$$
\begin{gathered}
N(f x, f y, k t) \leq \psi(N(g x, g y, t), N(f x, g x, t), N(f y, g y, t), \\
N(f x, g y, t), N(f y, g x, t))
\end{gathered}
$$

where $\varphi, \psi$ is a mapping from $[0,1]$ to $[0,1]$, which is upper semi-continuous, non-decreasing in each coordinate variable and such that

$$
\begin{aligned}
& \phi(1,1, t, 1, t) \geq t \\
& \phi(t, 1,1, t, t) \geq t
\end{aligned}
$$

and

$$
\begin{gathered}
\psi(1,1, t, 1, t) \leq t \\
\psi(t, 1,1, t, t) \leq t \text { where } t \in[0,1],
\end{gathered}
$$

(3.5) $g(X)$ is a closed subspace of $X$.

Then $f$ and $g$ have a unique common fixed point. 
Proof. In view of (3.1), there exist a sequence $\left\{x_{n}\right\}$ in $X$ such that $\lim _{\mathrm{n} \rightarrow \infty} f x_{n}=\lim _{\mathrm{n} \rightarrow \infty} g x_{n}=p$ for some $p \in X$. As $g(X)$ is a closed subspace of $X$, there is $u \in X$ such that $p=g u$. Therefore,

$$
\lim _{\mathrm{n} \rightarrow \infty} f x_{n}=p=g u=\lim _{\mathrm{n} \rightarrow \infty} g x_{n} .
$$

Now, we prove that $f u=g u$.

From (3.4) take $x=x_{n}, y=u$,

and

$$
\begin{gathered}
M\left(f x_{n}, f u, k t\right) \geq \varphi\left(M\left(g x_{n}, g u, t\right), M\left(f x_{n}, g x_{n}, t\right), M(f u, g u, t),\right. \\
\left.M\left(f x_{n}, g u, t\right), M\left(f u, g x_{n}, t\right)\right)
\end{gathered}
$$

$$
N\left(f x_{n}, f u, k t\right) \leq \psi\left(N\left(g x_{n}, g u, t\right), N\left(f x_{n}, g x_{n}, t\right), N(f u, g u, t), N\left(f x_{n}, g u, t\right), N\left(f u, g x_{n}, t\right)\right) .
$$

Taking the limit as $n \rightarrow \infty$, we get

$$
\begin{aligned}
M(g u, f u, k t) & \geq \varphi(M(g u, g u, t), M(g u, g u, t), M(f u, g u, t), M(g u, g u, t), M(f u, g u, t)) \\
& =\varphi(1,1, M(g u, f u, t), 1, M(g u, f u, t)) \geq M(g u, f u, t)
\end{aligned}
$$

and

$$
\begin{aligned}
N(g u, f u, k t)) \leq & \psi(N(g u, g u, t), N(g u, g u, t), N(f u, g u, t), \\
& N(g u, g u, t), N(f u, g u, t)) \\
= & \psi(0,0, N(g u, f u, t), 0, N(g u, f u, t)) \leq N(g u, u, t) .
\end{aligned}
$$

By using Lemma 2.1, we deduce that $f u=g u$. Denote $f u$ by $z$.

Therefore, $f u=g u=z$.

This shows that ' $u$ ' is coincident point of $f$ and $g$.

From weak compatibility of the mappings $f$ and $g$ it follows that $f g u=g f u$ This implies, $f z=g z$.

Now, we show that $z$ is the common fixed point of $f$ and $g$.

From (3.4) take $x=z, y=u$,

$$
\begin{aligned}
M(f z, z, t) & =M(f z, f u, t) \\
& \geq \varphi(M(g z, g u, t), M(f z, g z, t), M(f u, g u, t), M(f z, g u, t), M(f u, g z, t)), \\
& =\varphi(M(f z, z, t), 1,1, M(f z, z, t), M(z, f z, t)) \geq M(z, f z, t)
\end{aligned}
$$

and

$$
\begin{aligned}
N(f z, z, t) & =N(f z, f u, t) \\
& \leq \psi(N(g z, g u, t), N(f z, g z, t), N(f u, g u, t), N(f z, g u, t), N(f u, g z, t)), \\
& =\psi(N(f z, z, t), 0,0, N(f z, z, t), N(z, f z, t)) \leq N(f z, z, t) .
\end{aligned}
$$

By using Lemma 2.1, we deduce that, $f z=z=g z$ and thus we obtain that $z$ is a common fixed point of $f$ and $g$. 
Now, we prove the uniqueness of common fixed point of $f$ and $g$. If possible, let ' $a$ ' and ' $b$ ' be two common fixed points of $f$ and $g$.

Then by condition (3.4) take $x=a, y=b$ we get,

$M(f a, f b, k t) \geq \varphi(M(g a, g b, t), M(f a, g a, t), M(f b, g b, t), M(f a, g b, t), M(f b, g a, t))$

$M(a, b, k t) \geq \varphi(M(a, b, t), M(a, a, t), M(b, b, t), M(a, b, t), M(b, a, t))$

$\geq \varphi(M(a, b, t), 1,1, M(a, b, t), M(a, b, t))$

$M(a, b, k t) \geq M(a, b, t)$

and

$$
\begin{aligned}
N(f a, f b, k t) & \leq \psi(N(g a, g b, t), N(f a, g a, t), N(f y, g b, t), N(f a, g b, t), N(f b, g a, t)) \\
N(a, b, k t) & \leq \psi(N(a, b, t), N(a, a, t), N(b, b, t), N(a, b, t), N(b, a, t)) \\
& \leq \psi(N(a, b, t), 0,0, N(a, b, t), N(a, b, t)) \\
N(a, b, k t) & \leq \psi N(a, b, t) .
\end{aligned}
$$

Then by Lemma 2.1, we have $a=b$.

Therefore, the mappings $f$ and $g$ have a unique common fixed point.

This completes the proof.

\section{REFERENCES}

Aamri, M. and Moutawakil, D. El. (2002), 'Some new common fixed point theorems under strict contractive conditions', J. Math. Anal. Appl., 270, pp.181-188.

http://dx.doi.org/10.1016/S0022-247X(02)00059-8

Alaca, C., Turkoglu, D. and Yildiz C. (2006), 'Fixed points in Intuitionistic fuzzy metric spaces', Chaos, Solitons \& Fractals, 29, pp.

1073-1078. http://dx.doi.org/10.1016/j.chaos.2005.08.066

Atanassov, K.(1986), Intuitionistic Fuzzy sets, Fuzzy sets and system, 20, pp.87-96. http://dx.doi.org/10.1016/S0165-0114(86)80034-3

Coker, D.(1997), 'An introduction to Intuitionistic Fuzzy topological spaces', Fuzzy Sets and System, 88, pp. 81- 89. http://dx.doi.org/10.1016/S0165-0114(96)00076-0

Grorge, A. and Veeramani P.(1994), 'On some results in fuzzy metric spaces', Fuzzy Sets and Systems, 64, pp. 395- 399. http://dx.doi.org/10.1016/0165-0114(94)90162-7

Jungck, G.(1976), 'Commuting mappings and fixed points', Amer. Math. Monthly, 83, pp. 261263. http://dx.doi.org/10.2307/2318216

Kramosil, I. and Michalek, J.(1975), 'Fuzzy metric and Statistical metric spaces', Kybernetica, 11, pp.326-334.

Manro, S., Kumar, S. and Singh, S.(2010), 'Common fixed point theorems in intuitionistic fuzzy metric spaces', Applied Mathematics, 1, pp.510-514. http://dx.doi.org/10.4236/am.2010.16067

Manro, S., Bouharjera, H. and Singh, S.(2010), 'A common fixed point theorem in intuitionistic fuzzy metric space by using sub-compatible maps', Int. J. Contemp. Math. Sciences, 5(55), pp. $2699-2707$.
Common Fixed

Point Theorem

for Weakly

Compatible Maps in

Intuitionistic Fuzzy

Metric Spaces using

Implicit Relation 
Manro, S.

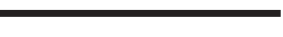

Manro, S., Bhatia, S. S., and Kumar, S. (2010), 'Common fixed point theorem for weakly compatible maps satisfying E.A. property in intuitionistic fuzzy metric spaces', Punjab University Journal of Mathematics, 42, pp. 51-56.

Manro, S., Kumar, S. and Bhatia, S.S.(2012), 'Common fixed point theorems in Intuitionistic fuzzy metric spaces using occasionally weakly compatible maps', J. Math. Comput. Sci., 2(2), pp.73-81.

Manro, S., Bhatia, S. S., and Kumar, S. (2012), 'Common fixed point theorems for weakly compatible maps satisfying common (E.A.) property in intuitionistic fuzzy metric spaces using implicit relation', Journal of Advanced Studies in Topology, 3(2), pp. 38-44.

Manro, S., Kumar, S., Kumar, S. and Bhatia, S. S.(2012), 'Common fixed point theorem in intuitionistic fuzzy metric spaces using common (E.A) property and implicit relation', Journal of Advanced Studies in Topology, 3(3), pp. 60-68.

Park, J. H. (2004), 'Intuitionistic fuzzy metric spaces', Chaos, Solitons \& Fractals, 22, pp.10391046. http://dx.doi.org/10.1016/j.chaos.2004.02.051

Park, J.S., Kwun, Y.C. and Park, J.H.(2005), 'A fixed point theorem in the Intuitionistic fuzzy metric spaces', Far East J. Math. Sci., 16, pp. 137-149.

Saadati R. and Park J.H.(2006), 'On the Intuitionistic fuzzy topological spaces', Chaos, Solitons \& Fractals, 27, pp. 331-344. http://dx.doi.org/10.1016/j.chaos.2005.03.019

Schweizer, B. and Sklar, A. (1960), 'Statistical metric spaces', Pacific J. Math., 10, pp. 313-334. http://dx.doi.org/10.2140/pjm.1960.10.673 http://dx.doi.org/10.2140/pjm.1960.10.313

Turkoglu D., Alaca C., Cho Y.J. and Yildiz C.(2006), 'Common fixed point theorems in Intuitionistic fuzzy metric spaces', J. Appl. Math. \& Computing, 22, pp. 411-424.

http://dx.doi.org/10.1007/BF02896489 